\title{
Le rôle d'une bibliothèque des sciences de la santé dans un Système Intégré pour la Gestion de l'Information Biomédicale (SIGIB)
}

The role of a health sciences library in a Système Intégré pour la Gestion de l'Information Biomédicale (SIGIB) El papel de una biblioteca de las ciencias de la salud en un Sistema Integrado por la Gestión de la Información Biomédica (SIGIB)

\section{Germain Chouinard}

Volume 41, numéro 2, avril-juin 1995

URI : https://id.erudit.org/iderudit/1033297ar

DOI : https://doi.org/10.7202/1033297ar

Aller au sommaire du numéro

Éditeur(s)

Association pour l'avancement des sciences et des techniques de la documentation (ASTED)

ISSN

0315-2340 (imprimé)

2291-8949 (numérique)

Découvrir la revue

Citer cet article

Chouinard, G. (1995). Le rôle d'une bibliothèque des sciences de la santé dans un Système Intégré pour la Gestion de l'Information Biomédicale (SIGIB).

Documentation et bibliothèques, 41(2), 89-93. https://doi.org/10.7202/1033297ar
Résumé de l'article

Les IAIMS, dont le sigle francisé SIGIB désigne un système intégré pour la gestion de l'information biomédicale, sont implantés de plus en plus dans les centres universitaires des sciences de la santé. Après avoir décrit

l'implantation de ce système à l'Université de Sherbrooke, l'auteur analyse son impact sur la clientèle, sur l'accomplissement des tâches et sur les besoins d'accès à la meilleure information, dans le cadre de la mise en place d'un réseau informatique.
Tous droits réservés (C) Association pour l'avancement des sciences et des techniques de la documentation (ASTED), 1995
Ce document est protégé par la loi sur le droit d'auteur. L'utilisation des services d’Érudit (y compris la reproduction) est assujettie à sa politique d'utilisation que vous pouvez consulter en ligne. 


\title{
Le rôle d'une bibliothèque des sciences de la santé dans un Système Intégré pour la Gestion de I'Information Biomédicale (SIGIB)
}

\author{
Germain Chouinard \\ Université de Sherbrooke
}

Les IAIMS, dont le sigle francisé SIGIB désigne un système intégré pour la gestion de l'information biomédicale, sont implantés de plus en plus dans les centres universitaires des sciences de la santé. Après avoir décrit l'implantation de ce système à l'Université de Sherbrooke, l'auteur analyse son impact sur la clientèle, sur l'accomplissement des tâches et sur les besoins d'accès à la meilleure information, dans le cadre de la mise en place d'un réseau informatique.

\section{The role of a health sciences library in a Système Intégré pour la Gestion de I'Information Biomédicale (SIGIB)}

Integrated Academic Information Management Systems (IAIMS) are increasingly present in universities that teach in the fields of the health sciences. Following a description of such a system at the Université de Sherbrooke, the author analyses its effects on users, on job descriptions, and on the need for access to better information.
El papel de una biblioteca de las ciencias de la salud en un Sistema Integrado por la Gestión de la Información Biomédica (SIGIB)

Los Integrated Academic Information Management System (IAIMS), cuya sigla francesa SIGIB designa un sistema integrado para la gestión de la información biomédica, son implantados más y más en los centros universitarios de las ciencias de la salud. Después de haber descrito la implantación de este sistema en la Universidad de Sherbrooke, el autor analiza su impacto sobre la clientela, sobre la realización de las tareas y sobre las necesidades de acceso a la mejorinformación, en el contexto delestablecimiento de una red informática.

\section{Un nouveau venu: le IAIMS}

Quand, le 16 octobre 1989, le docteur Gilles Pigeon est venu rencontrer les membres du comité de la bibliothèque pour leur expliquer ce qu'est un système IAIMS (se prononce ail aim ze), nous lui avons d'abord demandé de répéter le mot deux ou trois fois, et ensuite de nous en expliquer la signification. Le sigle IAIMS correspond à Integrated Academic Information Management System, dont la création remonte à quelques années.

Au début des années 80, l'Association of American Medical Colleges (AAMC) avait déjà proposé la création d'un système intégré d'information biomédicale. Par la suite, la National Library of Medicine des États-Unis mettra sur pied un programme d'aide afin de stimuler la planification stratégique en vue de l'implantation des IAIMS dans les centres universitaires des sciences de la santé. Les premiers program- mes subventionnés furent instaurés dans les universités Columbia, Georgetown, de Maryland et de l'Utah.

Les IAIMS sont d'abord issus d'un besoin créé par l'explosion des connaissances. L'information biomédicale connaît une croissance exponentielle, comme en témoigne l'augmentation phénoménale des revues scientifiques dans ce domaine. Les limites de la mémoire humaine ne permettent plus, même dans un domaine restreint, de suivre l'évolution des connaissances.

En même temps, les professeurs de médecine soulignaient l'importance de remplacer le programme traditionnel des études médicales basé sur la transmission des connaissances et la mémorisation, par une formation adaptée à la complexité et à l'évolution des connaissances visant la solution de problèmes et la gestion de l'information. La création des IAIMS est aussi liée à la complexité de plus en plus grande de la médecine et à la croissance exponentielle des coûts des soins de santé. Cette situation nécessite une intégration de multiples informations, qu'il s'agisse de systèmes experts d'aide au diagnostic, d'informations sur les médicaments pharmacocinétiques (coûts, interaction...), de protocoles de traitements, etc. Cette complexité nouvelle des outils de gestion: nécessité de mesurer l'efficacité et l'efficience, de modéliser les processus, d'évaluer les résultats et de corréler les différentes variables. II s'avère donc indispensable de disposer de moyens efficaces de stockage de l'information et de puissants instruments de communication afin que ces connaissances soient appliquées à l'enseignement, à la recherche, aux soins et à la gestion.

Depuis quelques années, nous assistons à la disponibilité croissante d'une technologie efficace, abordable et conviviale, 
en informatique, en communication ainsi que dans l'enseignement, l'évaluation ou la gestion, et ce phénomène libère des possibilités jusque là inaccessibles ou insoupçonnées.

Après l'exposé du Dr Gilles Pigeon, nous avons compris que ce IAIMS aurait un impact sur le fonctionnement de la bibliothèque des sciences de la santé. Mais il était trop tôt pour prédire notre avenir et amorcer un virage. Quatre ans plus tard, à l'automne de 1993, les autorités de la faculté de médecine ont émis l'énoncé de position suivant: «La Faculté de médecine de l'Université de Sherbrooke, ..., a comme projet de créer un IAIMS Sherbrooke». Destiné à favoriser la bonne exécution des quatre volets de la mission de notre complexe médical, ce projet d'un «IAIMS Sherbrooke» constitue un positionnement institutionnel stratégique pour le $X X I^{\text {e }}$. II repose sur des acquis solides (dossiers informatisés des patients, SNOMED, FAMUS,...), sur des forces vives internes ayant déjà conduit à l'innovation et au rayonnement de l'établissement (réforme des programmes de 1987, excellence en recherche, ...) et sur l'appui de plusieurs personnes ou groupes de l'Université de Sherbrooke.

Après quelques mois de travaux, le comité chargé d'étudier la faisabilité et les façons d'implanter un «IAIMS Sherbrooke» a présenté son rapport dans lequel on proposait la définition suivante d'un IAIMS, sigle francisé par SIGIB/Système Intégré pour la Gestion de l'Information Biomédicale. Cette définition décrit bien ce que doit être un SIGIB et, du même coup, nous oriente vers les objectifs à atteindre.

\section{La définition d'un SIGIB}

Un Système Intégré pourla Gestion de I'Information Biomédicale (SIGIB) se définit comme une infrastructure informatique qui permet aux personnes autorisées du secteur des sciences de la santé d'avoir, au moment et à l'endroit voulus, accès à la meilleure information possible afin d'accomplir d'une façon optimale leurs tâches d'enseignant, de chercheur, de clinicien ou de gestionnaire (et d'étudiant).

Quand on compare cette définition d'un SIGIB avec la mission et le mandat d'une bibliothèque universitaire, on a presque l'impression de lire le même texte:

\section{La mission}

La bibliothèque est un service qui a pour mission d'assurer à la communauté universitaire l'accès aux ressources documentaires requises aux fins de l'enseignement, de la recherche et des autres activités qui lui sont essentielles.

\section{Le mandat}

1. Développer une collection de documents propre à répondre aux besoins de la communauté universitaire en matière d'information documentaire et organisercette collection de façon à ce qu'elle soit exploitable (sélection, acquisition, mise en place, élagage)

2. Développer les liens appropriés avec les sources extérieures d'information documentaire, afin de répondre aux besoins de la communauté universitaire auxquels il serait impossible ou inopportun de satisfaire par la constitution d'une collection locale (coopération, prêt entre bibliothèques)

3. Permettre aux usagers une exploitation adéquate des ressources documentaires disponibles, qu'ils'agisse de la collection locale ou des ressources extérieures accessibles (prêt, entretien, contrôle, espaces, équipement)

4. Exercerun rôle d'expertise afin que les opérations s'effectuent en conformité avec les normes techniques appropriées.

La comparaison des textes permet de dégager les corollaires suivants: $1^{\circ} \mathrm{La}$ bibliothèque a un rôle important et essentiel à jouer dans un SIGIB, car dispenser l'information fait partie de sa mission propre. Il apparaît donc essentiel de participer à ce système; $2^{\circ}$ Cependant, selon ce même mandat, le domaine de la bibliothèque se situe dans le champ documentaire et bibliographique alors que le domaine d'information d'un SIGIB est beaucoup plus large. Cette précision devient importante lorsqu'il s'agit de partager les tâches et les ressources entre plusieurs partenaires (faculté, centre hospitalier, bibliothè- que, centre informatique, etc.) associés à la réalisation des mêmes objectifs poursuivis dans un SIGIB.

Impact sur le service de la bibliothèque

\section{Les usagers}

Selon la définition d'un SIGIB, les usagers sont «les personnes autorisées du secteur des sciences de la santé». Cette précision est importante en raison de la confidentialité et de la sécurité d'accès reliées à certaines informations touchant la santé, comme le dossier médical, par exemple. Cependant, la bibliothèque des sciences de la santé n'a pas d'information confidentielle à protéger, hormis ce qui concerne la vie privée des utilisateurs. De plus, les services de la bibliothèque ne sont pas limités aux niveaux d'autorisations accordées aux divers groupes de professionnels de la santé dans l'exercice de leur fonction. Donc, l'implantation d'un SIGIB à Sherbrooke n'a pas modifié le profil de nos usagers.

La bibliothèque dessert la faculté de médecine, le Centre hospitalier universitaire et le Centre de recherche clinique. Nos usagers sont d'abord les professeurs (cliniciens et chercheurs) de la faculté, les étudiants de la faculté (en médecine, en sciences infirmières et aux études avancées) et le personnel des trois établissements que nous desservons (administrateurs, infirmiers, techniciens et professionnels, etc...). Bientôt un quatrième se joindra aux trois premiers: l'Institut de pharmacologie. Nous desservons aussi les autres facultés et bibliothèques de la communauté universitaire qui utilisent parfois les collections et les services de la Bibliothèque des sciences de la santé et qui tirent avantage de sa participation à un SIGIB mis en place pour répondre aux besoins de l'enseignement médical. Ce qui s'avère utile à un groupe en particulier l'est également à toute la communauté.

\section{L'accomplissement des tâches}

Le SIGIB n'a pas modifié la liste des tâches d'enseignement et de recherche pour lesquelles la bibliothèque est mandatée. Mais la documentation publicitaire de la faculté insiste sur l'auto-apprentissage. L'étudiant doit rechercher lui-même l'information 
documentaire et bibliographique dont il a besoin. Cette nécessité entraîne forcément, pour la bibliothèque, des changements dans la façon de dispenser des services.

L'utilisation des salles de séminaires pour le travail en équipe s'est accrue considérablement. La bibliothèque ne suffit pas à la demande même si nous offrons tout ce qui est disponible. La Faculté a dû elle-même augmenter le nombre de salles pour petits groupes. Le nombre d'ouvrages en réserve a été augmenté en nombre d'exemplaires d'un même titre et en nombre de titres. En 1993-94 nous avons évalué que le prêt des ouvrages de la réserve constituait $75 \%$ de l'utilisation totale des monographies.

Plusieurs motifs militent en faveur de ce virage "pédagogique», le plus important de tous étant l'accroissement du nombre des publications biomédicales et l'explosion des connaissances. La méthode d'enseignement qui ne tiendrait pas compte de ce flot continuel d'information deviendrait vite dépassée par la quantité de l'information: 10000 revues dont 4000 indexées dans Medline plus 75000 nouveaux articles de périodiques publiés à chaque mois dont 30000 dans Medline uniquement et la provenance multiple des informations: des laboratoires et des institutions d'enseignement de plus de 70 pays publient les résultats de leur recherche dans Medline.

La bibliothèque des sciences de la santé d'un SIGIB doit planifier son développement en tenant compte obligatoirement du fait que l'apprentissage individuel et la formation personnelle continue des étudiants en sciences de la santé sont devenus les moyens pédagogiques privilégiés de leur formation. Cette tendance à l'auto-apprentissage est exigeante pour la bibliothèque. Outre le besoin plus grand de faire usage des ressources de la bibliothèque, il faut retenir la nécessité d'une formation documentaire qui facilite l'accès aux richesses de la bibliothèque. Cette activité a augmenté la part de temps que le personnel de la bibliothèque doit consacrer à la formation de la clientèle. La demande de formation est beaucoup plus grande que ce qu'il est possible d'offrir, faute de personnel, faute aussi de service semblable ailleurs ou faute d'avoir bien défini les responsabilités de formation. Dans notre milieu où faculté et hôpital sont dans le même édifice, notre bibliothèque doit aussi jouer un rôle additionnel, celui d'informer le personnel médical et paramédical dans le domaine des soins de santé. II est évident que dans un SIGIB qui insiste sur la qualité des soins de santé, la demande pour une information de qualité, véridique, accessible, récente et diversifiée s'accroît considérablement.

\section{Accès à l'information la meilleure}

Dans l'expression «l'information la meilleure», le premier élément à considérer est l'information elle-même. En effet, le concept d'information peut varier grandement selon la personne qui manifeste un besoin d'information. Celle-là est constituée d'éléments divers: données, renseignements et connaissances. Ainsi, la température d'un patient prise à toutes les heures constitue des données. Et cet ensemble de données nous renseigne sur l'état de la température du patient. $\mathrm{Ce}$ renseignement, associé à d'autres renseignements sur le patient, nous conduira à l'identification de la maladie. Le rôle de la bibliothèque dans cette quête de l'information consiste à fournir non pas les données confidentielles contenues dans les dossiers médicaux des patients, mais à donner accès aux diverses données sur les maladies à soigner, sur les soins à dispenser et sur les recherches effectuées dans ce domaine, toutes ces informations étant publiées sous divers supports dans différents types de publications.

Le deuxième élément à considérer dans l'expression mentionnée plus haut concerne la qualité de l'information. Que faut-il sous-entendre ici dans une société où l'on parle de plus en plus de qualité totale? L'information "la meilleure» est intègre et digne de confiance. Des données et des renseignements inexacts ne peuvent pas conduire à la connaissance requise pour exercer la médecine, ni à l'avancement des connaissances en général. L'information «la meilleure» est accessible. Elle doit être disponible sur place, ou provenir rapidement de l'extérieur parce qu'elle est requise maintenant.
L'information «la meilleure» est à jour et récente. Dans le secteur de la santé, beaucoup de recherches et de découvertes s'effectuent quotidiennement. Les périodiques en publient régulièrement les résultats. II est devenu impensable de pratiquer la médecine sans consulter fréquemment les études scientifiques les plus récentes. Ces changements rapides dans les connaissances biomédicales ont entraîné des changements dans la façon de former les médecins. Le besoin des médecins de vérifier régulièrement ce qui a été rapporté dans la documentation, sur les maladies et leur traitement, les a incités à publier les résultats de leurs propres découvertes et, du même coup, à contribuer à faire augmenter le nombre de publications scientifiques.

L'information «la meilleure» est aussi diversifiée car elle implique la variété des sources: banques de données bibliographiques portant sur des sujets biomédicaux multiples, catalogues des ouvrages disponibles localement et dans d'autres bibliothèques régionales, nationales et internationales. On constate de plus en plus une diversité de sujets et de supports, ainsi qu'une diversité des moyens d'accès aux contenus.

Ces éléments imposent à la bibliothèque d'un SIGIB une contrainte importante si cette bibliothèque veut continuer à dispenser l'information la meilleure, la plus intègre, la plus récente, selon des sources diversifiées et selon des techniques variées. II faut obligatoirement faire des choix et surtout implanter le système par étapes, selon les priorités du milieu. Afin de répondre aux besoins de notre bibliothèque, nous avons acheté les banques Medline, Current Contents et le compendium des produits et spécialités pharmaceutiques (Axis-CPS). Nous offrons aussi accès à plus de 50 autres banques sur disques compacts disponibles dans les autres bibliothèques de l'Université de Sherbrooke, dont une vingtaine présentent un intérêt biomédical. Le catalogue centralisé donne aussi un accès aux collections pour l'ensemble des bibliothèques de l'Université. Pour les disciplines où nous n'avons pas de banques de données sur place, nous continuons d'offrir les services de consultation en ligne (Dialog, CD Plus, etc.). 


\section{L'accès à l'information à l'endroit et} au moment voulus

Autrefois, les usagers qui avaient besoin d'information devaient obligatoirement se rendre à la bibliothèque. Dans notre ère de technologie, les utilisateurs des services de la bibliothèque exigent de plus en plus que la bibliothèque se rende jusqu'à eux, là où ils sont. Cet «endroit voulu» varie d'un individu à l'autre. Les endroits les plus fréquemment identifiés (en dehors de la bibliothèque qui reste un endroit privilégié pour la consultation) sont le bureau, le département de la faculté ou la clinique, le domicile et l'hôpital affilié.

L'accès à la documentation «au moment voulu» signifie que l'usager dispose, 24 heures par jour, de certains services de la bibliothèque tels que les banques de données (Medline et Current Contents), le catalogue de la bibliothèque (Sibus) et le compendium des produits pharmaceutiques (Axis-CPS). Présentement, nous offrons ces trois catégories d'information. On sollicite de plus en plus un accès au texte intégral des principales revues biomédicales ainsi qu'à d'autres banques de données. Éventuellement, ces services seront-ils accessibles localement ou à distance? Tout dépendra des développements de la technologie, des services offerts par les fournisseurs et des coûts d'achat ou d'abonnement. Récemment, nous avons analysé le système ADONIS qui offre plus de 600 revues biomédicales en texte intégral sur disque compact, mais qui ne s'est pas révélé le produit répondant le mieux à nos besoins.

L'accès à la documentation «au moment voulu» signifie, durant les heures d'ouverture de la bibliothèque, avoir un accès rapide à ce qui n'est pas disponible sur place. II faut alors utiliser les technologies modernes comme le courrier électronique, le télécopieur, INTERNET, l'informatisation du prêt entre bibliothèques (Romulus, Peb-UQ), et bientôt l'imagerie électronique (Ariel) et Docline. Quant aux. périodiques, en plus de ceux disponibles en bibliothèque, nous avons également identifié ceux disponibles dans la faculté (197 nouveaux titres uniques), dans les autres bibliothèques de l'Université de Sherbrooke (284 titres uniques indexés dans Medline) et dans les hôpitaux affiliés de la région (15 titres environ). Nous comp- tons également évaluer les collections des autres bibliothèques de la région. Tous ces titres disponibles dans la bibliothèque ou à quelques kilomètres autour du campus sont ou seront inscrits dans nos banques de données afin d'informer immédiatement l'usager de la disponibilité des publications.

\section{Mise en place d'un réseau informatique}

Afin de répondre aux besoins identifiés plus haut, un ensemble de réseaux informatiques possède l'infrastructure et la flexibilité nécessaires. À la fin des années 80 , le Centre hospitalier universitaire

\section{Réseau MED-Plus \\ Blbllothèque des sciences de la santé Faculté de médecine \\ A U UNIVERSITE DE SHERBROOKE}

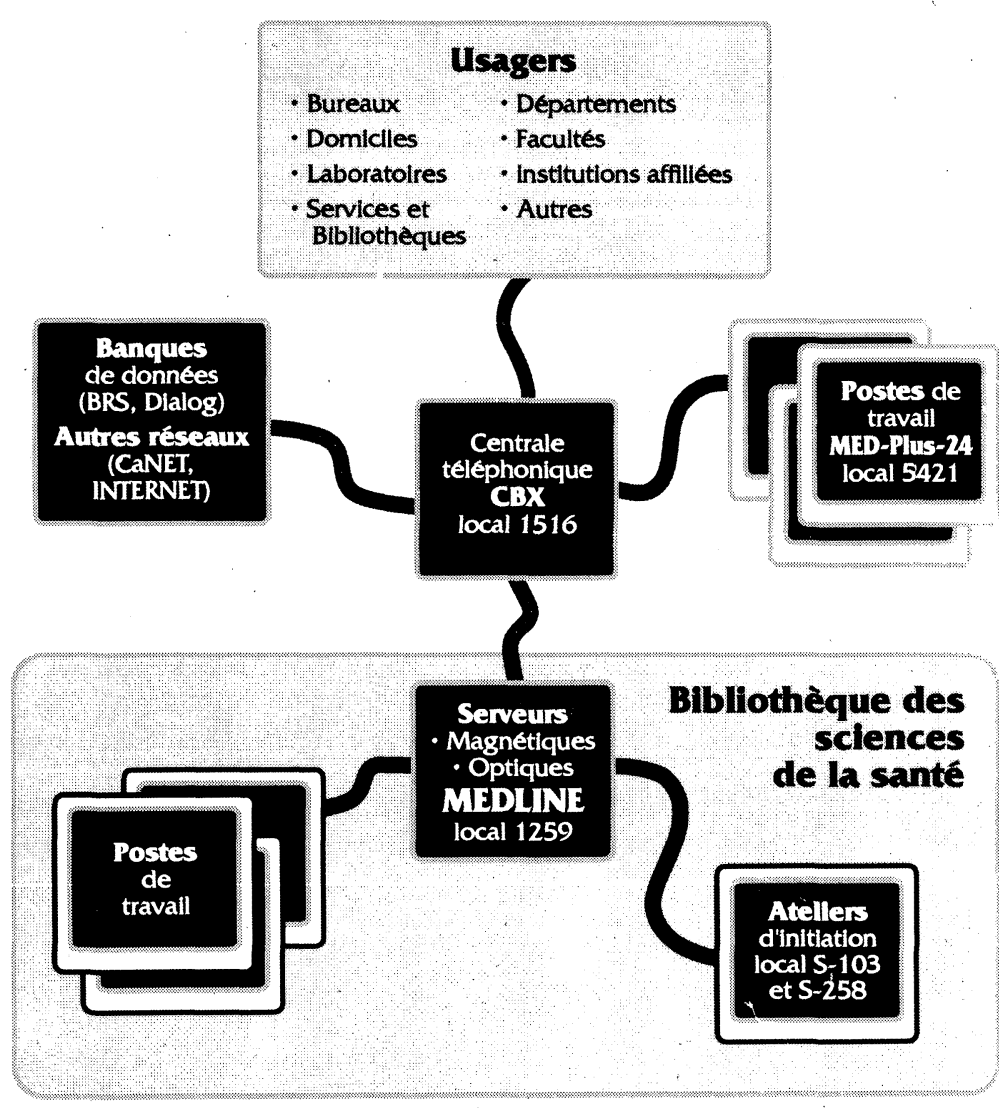


de Sherbrooke implantait son réseau clinique (Ariane) avec plus de 600 terminaux installés à proximité des lits des patients et aux endroits propices à dispenser les soins.

En 1993, le Service des bibliothèques de l'Université de Sherbrooke mettait en place le réseau Med-Plus, avec Medline accessible 24 heures par jour à seize usagers simultanément grâce à six postes de travail, à douze modems et aux câbles Ethernet dans les locaux de la bibliothèque et dans un local à l'extérieur de la bibliothèque. Durant la même période, le catalogue de nos collections devenait aussi accessible sur le réseau INTERNET et le Service informatique reliait les deux campus universitaires par un lien Ethernet/ micro-ondes. Les autres bibliothèques de I'Université de Sherbrooke achetaient des bànques de données sur disques compacts chargés sur des serveurs Novell accessibles sur l'Ethernet local.

En 1994, la faculté de médecine (médecine et sciences infirmières) reliait plus de 550 bureaux et laboratoires à I'Ethernet local et à l'INTERNET, grâce au lien micro-ondes. En 1994-95, la bibliothèque, la clinique et la faculté travaillent à mettre au point des postes à guichet unique Windows qui donneront à la fois un accès aux banques de la bibliothèque (Med-Plus), aux dossiers des patients (Ariane), au catalogue de la bibliothèque (Sibus) et au compendium des produits pharmaceutiques (Axis). On envisage de mettre en place 12 postes dédiés dans les départements cliniques et de configurer les postes des professeurs selon leurs besoins respectifs. Avec le temps, d'autres banques d'information documentaire et bibliographique (Scientific American, Disco Test, Gene Bank, etc.) seront disponibles à distance.

\section{Conclusion}

Certains diront que tout cela est déjà disponible ailleurs sans pour autant faire partie d'un SIGIB. II est évident que tous

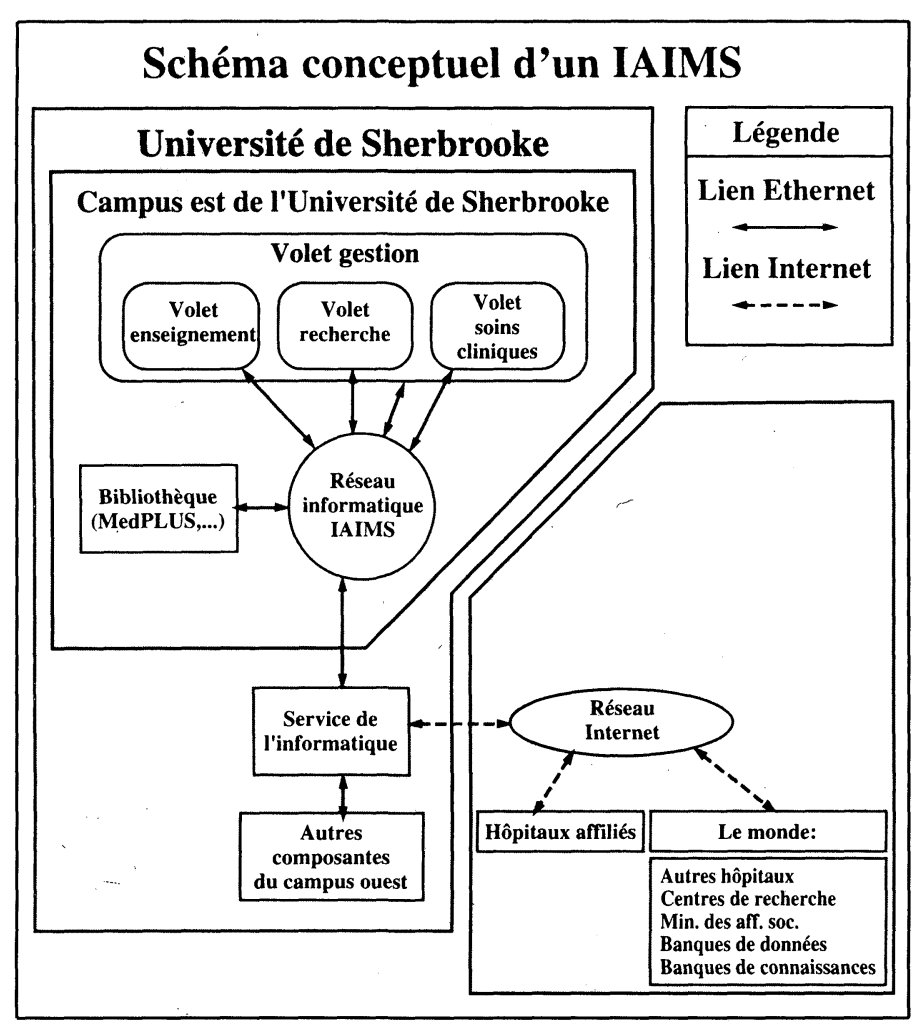

ces développements se font également dans d'autres bibliothèques. Cependant, les bibliothèques de la santé qui font partie d'un SIGIB deviennent en quelque sorte les laboratoires de recherche de la bibliothéconomie, où on expérimente de façon active et intensive les nouveaux moyens d'informer et de former ceux qui travaillent à cette cause excellente qu'est la santé de l'être humain.

En tant que spécialistes du transfert de l'information documentaire du domaine des sciences de la santé, nous avons le privilège d'utiliser et de tester aujourd'hui même ces nouveaux supports de l'information, ces nouveaux moyens de communications et ces nouvelles technologies. Même si nous ne sommes pas seuls à exécuter ces tâches, nous devons nous maintenir dans le peloton de tête à cause de l'importance des enjeux.

\section{Bibliographie}

Broering, NaomiC.Creating a Knowledge Network Dahlgren Memorial Library (Health Sciences Library), Georgetown University Medical Center, 1993.

Chassé, Jules. Lesréseaux d' informationaccessibles par la bibliothèque. Service des bibliothèques, Université de Sherbrooke,Janvier 1994.

Lorenzi, Nancy M. "A decade of IAIMS (Symposium).» Bulletin of the Medical Library Association 80, no. 3 (July 1992).

Projet Sigibus (IAIMS - Sherbrooke). Faculté de médecine, Université de Sherbrooke, Juin 1994.

Thibault, Richard. "IAIMS.» Le Bulletin de la Faculté de médecine, Université de Sherbrooke 6 , $\mathrm{n}^{\circ} 3$ (juin 1994) 